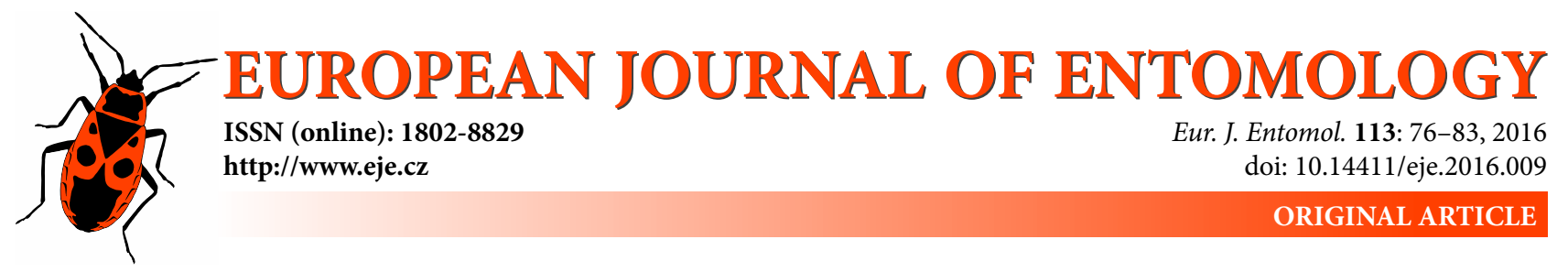

\title{
Identification and expression pattern analysis of chemosensory receptor genes in the Macrocentrus cingulum (Hymenoptera: Braconidae) antennae
}

\author{
Tofael AHMED ${ }^{1,2}$, TIAN-TAO ZHANG ${ }^{1, *}$, ZHen-YING WANG $^{1, *}$, KANG-LAI HE $^{1}$ and Shu-XIONg BAI ${ }^{1}$ \\ ${ }^{1}$ State Key Laboratory for the Biology of the Plant Diseases and Insect Pests, Institute of Plant Protection, Chinese Academy of \\ Agricultural Sciences, Beijing 100193, China; e-mails: tofaela@yahoo.com, zhtiantao@163.com, zywang@ippcaas.cn, \\ klhe@ippcaas.cn, sxbai@ippcaas.cn \\ ${ }^{2}$ Bangladesh Sugarcane Research Institute, Ishurdi-6620, Pabna, Bangladesh
}

Key words. Hymenoptera, Braconidae, Macrocentrus cingulum, cDNA library, odorant and ionotropic receptors, expression pattern

\begin{abstract}
Macrocentrus cingulum is an important polyembryonic endoparasitic wasp that attacks larvae of the Asian corn borer, Ostrinia furnacalis (Guenée) and the European corn borer, O. nubilalis (Hübner). Parasitoids use antennae as the main sensory organ to recognize herbivore-induced plant volatiles as host searching cues. The antennal olfaction proteins, odorant receptors (ORs) and ionotropic receptors (IRs) are involved in olfactory signal transduction pathway as a sensory neuron response. In the present study, we constructed a cDNA library from the male and female antennae for identifying the olfaction-related genes in $M$. cingulum. For that, we sequenced 3160 unique gene sequences and annotated them with gene ontology (GO), cluster of orthologous groups of proteins (COG), and KEGG ontology (KO). Through the homology search, we identified 9 odorant receptors (ORs), 3 ionotropic receptors (IRs) and 1 odorant binding protein (OBP) genes from the cDNA library sequences. Additionally, the expression patterns of these ORs and IRs in different tissues (antennae, heads, thoraxes, abdomens, and legs) were demonstrated by RT-PCR. The qualitative gene expression analyses showed that most of the OR genes were more highly expressed in female than male antennae; whereas IRs, unlike ORs, were more expressed in various male than females tissues. We are the first to report ORs and IRs in M. cingulum, which should help in deciphering the molecular basis of olfaction system in this wasp.
\end{abstract}

\section{INTRODUCTION}

Olfaction is an important sensory assessment of the environment, which is crucial to survival and reproduction, and which directly regulates the behavior of organisms. In insects, chemosensory receptors sense the alarm signals, host-plant cues and conspecific pheromones (Olivier et al., 2011). Volatile compounds are detected by olfactory receptors expressed in the dendritic membranes of olfactory sensory neurones (OSNs) that are housed in sensory hairs called olfactory sensilla, located on antennae, maxillary palps and even ovipositor sheaths (Bengtsson et al., 2012; Andersson et al., 2013). Olfactory receptors provide information on the chemical quality such as food sources or oviposition sites and also are used in intraspecific communication via pheromones (Grosse-Wilde et al., 2011). In almost all insect species, repertoires of several to several hundred of highly divergent odorant receptors (ORs) are responsible for detecting the myriad of volatile chemical signals in the environment ( $\mathrm{Su}$ et al., 2009). Insect ORs are seven-transmembrane domain receptors with inverted membrane topology compared to vertebrate ORs, to which they are unrelated (Benton et al., 2006). ORs function with a conserved co-receptor protein named ORco, acting as an ion channel (Larsson et al., 2004). The gustatory receptors (GRs) are mostly expressed in gustatory receptor neurons (Anderson et al., 2013) and are structurally related to ORs, ionotropic receptors (IRs), and ionotropic glutamate receptors (Benton et al., 2009).

The number of insect OR genes varies from species to species due to their divergence with most insects having 50 to 200 such genes (Bengtsson et al., 2012). The first OR genes, identified with a genomic analysis of Drosophila melanogaster encoded seven transmembrane domains that were largely expressed in morphological and functional

\footnotetext{
* Corresponding authors. Present address: Institute of Plant Protection, Chinese Academy of Agricultural Sciences, No. 2 West Yuanmingyuan Road, Beijing 100193, China; e-mails: zhtiantao@163.com, zywang@ippcaas.cn.
} 
types of olfactory sensilla, especially trichoid and basiconic sensilla (Clyne et al., 1999; Gao \& Chess, 1999; Vosshall et al., 1999; Couto et al., 2005). Insects respond to a variety of chemical cues with their highly diverse receptors (Howlett et al., 2012). In addition to receptor genes, other multigene families encode proteins such as odorant binding proteins (OBPs) and chemosensory proteins (CSPs), which are gene families with critical roles in taste and olfaction (Andersson et al., 2013). The small, water soluble OBPs within the lymphatic cavity of olfactory sensilla that facilitate ligand binding to ORs (Gong et al., 2009) but CSPs may help mediate binding between ligands and receptors (Gong et al., 2007).

Insect ionotropic receptor (IR) genes were discovered in D. melanogaster by bioinformatic analyses for insectspecific genes with enriched expression in OSNs (Benton et al., 2009). Apparently, IRs are related to ionotropic glutamate receptors (iGlurs), which are involved in synaptic signal transduction in both vertebrates and invertebrates (Bengtsson et al., 2012). IRs have atypical binding domains that are more conserved than ORs; thus it has been possible to identify several paralogous lineages among insects (Bengtsson et al., 2012). IR-induced responses appear to be conferred by assemblies of variable subunits in a heteromeric receptor, as up to five different IRs can be co-expressed in a single OSN (Benton et al., 2009). A functional complex is formed by two or more subunits of IRs, and its involved as a specific odor receptor (Bengtsson et al., 2012). Broadly expressed IR25a and IR8a in D. melanogaster function as co-receptors, similar to $O R 83 b$ in OR complexes (Benton et al., 2009; Croset et al., 2010).

Olfaction is critical for insect survival as it plays an important role in locating food sources, mating partner, oviposition site, and prey or host (Liu et al., 2012; Nishimura et al., 2012; Ahmed et al., 2013). Parasitoid effectiveness depends on their searching ability to locate the host (Nordlund et al., 1988). Macrocentrus cingulum Brischke (Hymenoptera: Braconidae) is a polyembryonic endoparasitoid of the Asian corn borer, Ostrinia furnacalis (Guenée) (Lepidoptera: Crambidae) and the European corn borer, O. nubilalis (Hübner) (Edwards \& Hopper, 1999; Hu et al., 2003). The native range of $M$. cingulum is distributed across Europe and throughout Asia, including Japan, Korea and China (Watanabe, 1967). Parasitoids use herbivore induced plant volatiles (HIPVs) and green leaf volatiles (GLVs) from infested plants by host insect as chemical cues (Ochieng et al., 2000; Shiojiri et al., 2000). M. cingulum has evolved an efficient olfaction system to locate its host (Ahmed et al., 2013). Female M. cingulum use host larval frass in stalk tunnels as host-searching cues (Parker, 1931). We constructed a cDNA library from the male and female antennae of $M$. cingulum and used it to identify olfaction-related genes and study olfactory signal transduction mechanisms. Sex-specific expression patterns of various tissues were analyzed for identified OR and IR genes.

\section{MATERIALS AND METHODS}

\section{Insects}

Specimens of $M$. cingulum were obtained from $O$. furnacalis larvae living on corn plants at the Langfang Experiment Station of the Institute of Plant Protection, Chinese Academy of Agricultural Sciences, China. The parasitoids emerged as mature larvae from the host larvae and pupated inside the silken cocoon. Adult parasitoid wasps were fed with $20 \%$ honey solution. A laboratory colony was maintained on host larvae of $O$. furnacalis that were reared on an artificial diet as described by Zhou et al. (1980). They were maintained in a room at $25^{\circ} \mathrm{C}$ with a $16 \mathrm{~L}: 8 \mathrm{D}$ regime (Ahmed et al., 2013). Antennae, heads with maxillary palps (excluding antennae), legs, thorax, and abdomen of female and male individuals were dissected 1-3 days after eclosion and immediately frozen in liquid nitrogen, then stored at $-80^{\circ} \mathrm{C}$ until RNA extraction.

\section{Extraction of total RNA}

The frozen antennae (100 pairs of each sex) or other tissues were homogenized with a liquid nitrogen cooled mortar and ground with a pestle into very fine dust. Homogenized tissues were covered with $1 \mathrm{~mL}$ of TriZol reagent (Invitrogen, Carlsbad, CA, USA) and total RNA were extracted following the manufacturer's instructions. After extraction, RNA integrity was verified by $1 \%$ agarose gel electrophoresis and quantity was assessed with a Nanodrop ND-1000 spectrophotometer (NanoDrop products, Wilmington, DE, USA).

\section{Construction of cDNA library, gene identification and functional annotation}

For the synthesis of the first-strand cDNA we used $1 \mu \mathrm{g}$ of total RNA. The antennal cDNA library was constructed using the InFusion ${ }^{\circledR}$ SMARTer ${ }^{\mathrm{TM}}$ Directional cDNA Library Construction Kit (Clontech, Mountain, CA, USA), according to manufacturer's instructions. Sizes of cDNA inserts were determined using PCR. A pair of sequence-specific primers was designed based on the sequence of the pDNR-LIB plasmid. The clones that contained the cDNA insert longer than $400 \mathrm{bp}$ were chosen and sequenced using ABI3730 sequencer (SANGON Sequencing Service, Shanghai, China). The nucleotide sequences were searched against the NCBI non-redundant protein database on a local server using the National Center for Biotechnology Information (NCBI) BLASTALL program (Altschul et al., 1997). Gene ontology (GO) annotation was performed by using Blast2GO (GO association done by a BLASTX against the NCBI NR database) (Conesa et al., 2005; Götz et al., 2008). Olfactory related genes were identified by homology searching. Duplicate genes were found by the alignment module of DNAMAN software (Lynnon Biosoft, San Ramon, CA, USA); MEGA 4.0 software was used to construct phylogenetic trees (Tamura et al., 2007). Also, unique gene sequences (unigenes) were aligned to the COG database (database of Clusters of Orthologous Groups of proteins; Tatusov et al., 2000) to find homologous genes, which facilitated more accurate function annotations. In addition, we used Kyoto Encyclopedia of Genes and Genomes (KEGG; Kanehisa Laboratories, Japan) ontology (KO) enrichment analyses to investigate biological functions.

\section{RT-PCR for expression analysis}

To explore the expression of the ORs/IRs identified from the antennal cDNA library analyses and to compare the differential expression pattern between the sexes, RT-PCR was conducted with cDNAs prepared from different tissues of male and female wasps. Independent triplicate individual samples of total RNA were isolated from the above mentioned tissues and correspond- 


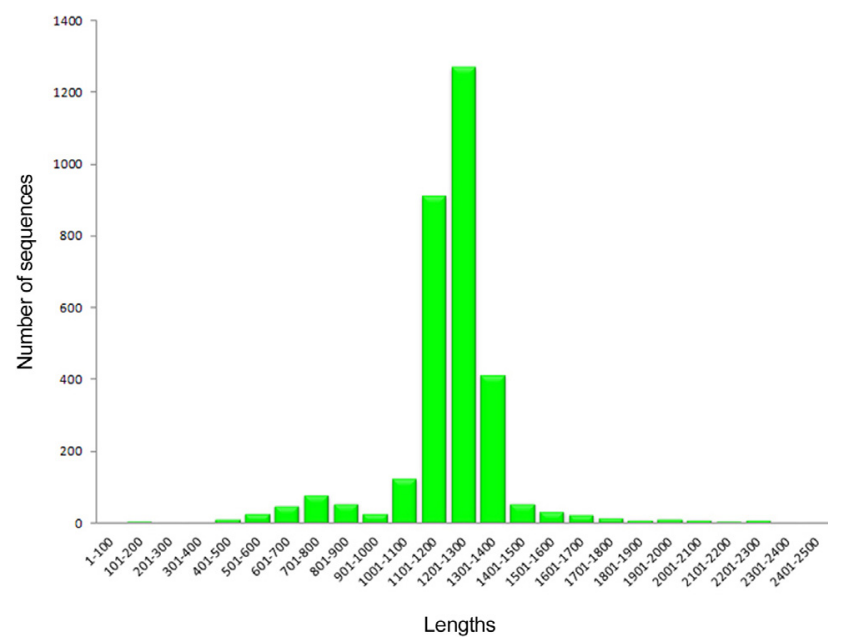

Fig. 1. Distribution of unigene sequences of cDNA library of Macrocentrus cingulum are shown in different length interval.

ing cDNAs were synthesized using the RT-for-PCR kit (Clontech, Mountain View, CA, USA) following the kit manual. $\beta$-actin was used as reference gene (accession no. EU585777.1) and it was used to select the cDNA templates on the PCR equipment. Primers that were designed manually or with the Primer 5 tool (http://frodo.wi.mit.edu/primer5/) to yield 100-200 bp product sequences are available in Table 1 . Individual PCR reactions were repeated three times; controls consisted of no template PCR. The PCR conditions consisted of an initial 3-min step at $94^{\circ} \mathrm{C}, 30 \mathrm{cy}$ cles of $94^{\circ} \mathrm{C}$ for $30 \mathrm{~s}, 56,57$ or $59^{\circ} \mathrm{C}$ (depending on primers) for $30 \mathrm{~s}$ and $72^{\circ} \mathrm{C}$ for $3 \mathrm{~min}$ and finally 10 -min step at $72^{\circ} \mathrm{C}$. Products

Table 1. Primers for RT- PCR expression pattern analysis of ORs and IRs in Macrocentrus cingulum.

\begin{tabular}{llc}
\hline Primer name & \multicolumn{1}{c}{ Primer (5'to $\left.{ }^{\prime}{ }^{\prime}\right)$} & $\begin{array}{c}\text { Predicted } \\
\text { Tm }\left({ }^{\circ} \mathrm{C}\right)\end{array}$ \\
\hline McinOR1-Forward & CTGCTGCTAGGCAAGGATGAAT & 60.1 \\
McinOR1-Reverse & TGCCTCAGCGACAAGTGTTG & 59.8 \\
McinOR2-Forward & CCAATGCCTGCGCAAAAG & 57.3 \\
McinOR2-Reverse & GACTGCAGCATGTCGACTAGTTTC & 62.0 \\
McinOR3-Forward & GGCCAAAACAACAGACAGAACTC & 60.2 \\
McinOR3-Reverse & GCAGTAGCCAGGACTGAGCTTATAT & 62.0 \\
McinOR4-Forward & TGAGTGAACGATTTCCATTTCC & 56.6 \\
McinOR4-Reverse & TCTTGGGTTGGAAAGTCGTA & 58.0 \\
McinOR5-Forward & TGGACGACGGGTTTGA & 57.0 \\
McinOR5-Reverse & CAACACAACGCTCACGAACAT & 58.0 \\
McinOR6-Forward & TCCGTGAGCCTGGTGATTATT & 58.0 \\
McinOR6-Reverse & GCGGTAAAGGTGCAACATT & 57.8 \\
McinOR7-Forward & GCCTATATTGGCGGTGACGTT & 60.0 \\
McinOR7-Reverse & GCAGTTGAATTGGCCACATAC & 58.2 \\
McinOR8-Forward & TGAGCAGTCATGCAGTCTGATG & 60.1 \\
McinOR8-Reverse & ATCCCAGGACGCAGAATGTAAG & 60.1 \\
McinOR9-Forward & CGGTGCTTTACTACTCGCTGTATC & 62.0 \\
McinOR9-Reverse & AAACTGCAACTCCCGTTGGT & 57.8 \\
McinIR1-Forward & CGTCACGGCGACAAAGATT & 57.6 \\
McinIR1-Reverse & AAGCGCGGCATTGAAGAG & 57.3 \\
McinIR2-Forward & GCGAAGAGCTTCCCCAATT & 57.8 \\
McinIR2-Reverse & ACGTCCGATCAATCTCGTCAT & 58.2 \\
McinIR3-Forward & TCCGATTTCACGCATCAGTTC & 58.0 \\
McinIR3-Reverse & CTCGTGGTGGGATCACA & 59.6 \\
Mcinßactin-Forward & TATGTCGCCCTGGACTTTG & 57.6 \\
Mcinßactin-Reverse & TTGGTGATGATTCCGTGCT & 55.4 \\
\hline & &
\end{tabular}

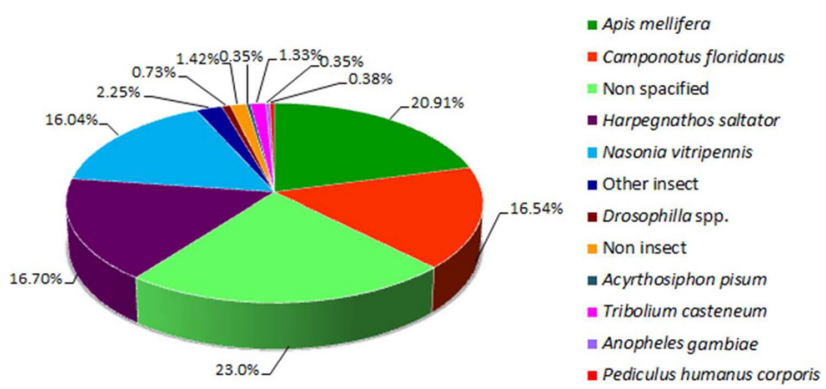

Fig. 2. Species distribution of homology search with the Macrocentrus cingulum unigenes against the $\mathrm{Nr}$ database. The species distribution is shown as a percentage of the total homologous sequences in the NCBI Nr protein database with an $E$-value $<10^{-5}$.

were analyzed on a $1 \%$ agarose gel and visualized after staining with ethidium bromide.

\section{RESULTS}

\section{Antennal cDNA library of $\boldsymbol{M}$. cingulum}

The antennal cDNA library was constructed successfully from female and male $M$. cingulum. Titer of this library was $1.6 \times 10^{6} \mathrm{pfu} / \mathrm{mL}$; indicating a sufficient number of genes that were expressed. Genes, longer than $\sim 0.4 \mathrm{~kb}$ were selected and sequenced from the library, which resulted in 3160 high quality unigenes for further analysis. Most of the unigenes ranged from $1000 \mathrm{bp}$ to $1400 \mathrm{bp}$ with a mean length of $1203 \mathrm{bp}$. The unigene length distributions are shown in Fig. 1.

\section{Annotation of predicted proteins}

To annotate the $M$. cingulum antennal unigenes, distinct sequences were searched by BLASTx against the NCBI non-redundant protein database $(\mathrm{Nr})$ with a cut-off $E$-value of $10^{-5}$. Of the total 3160 unigenes, $77.00 \%$ matched with known gene sequences; the other $23.00 \%$ were not similar to any sequence in the $\mathrm{Nr}$ database information at presents. The top BLAST hit results for each unique sequence are shown in Fig. 2. The unigenes from $M$. cingulum revealed that the highest number of genes, 661 (20.91\%) are homologus to the honey bee (Apis mellifera) followed by 528 (16.70\%) from the ponerine ant (Harpegnathos saltator), 523 (16.54\%) from the Florida carpenter ant (Camponotus floridanus) and 507 (16.04\%) from the jewel wasp (Nasonia vitripennis).

\section{Functional annotation by GO, COG, and KEGG}

International recommended gene function classification system of Gene Ontology (GO) covers three domains: biological process, cellular component and molecular function. The GO unigene annotation (WEGO software; Ye et al., 2006) allowed us to predict gene functions of both sexes of this species at the molecular level. The GO annotated unigenes were categorized into 46 functional groups. "Cell" and "Cellular process" are the two largest groups; containing 839 and 791 unigenes, respectively. Three smaller groups: viral reproduction, nucleic acid binding transcription activity and structural molecule activity; each had only one unigene (Fig. 3). 


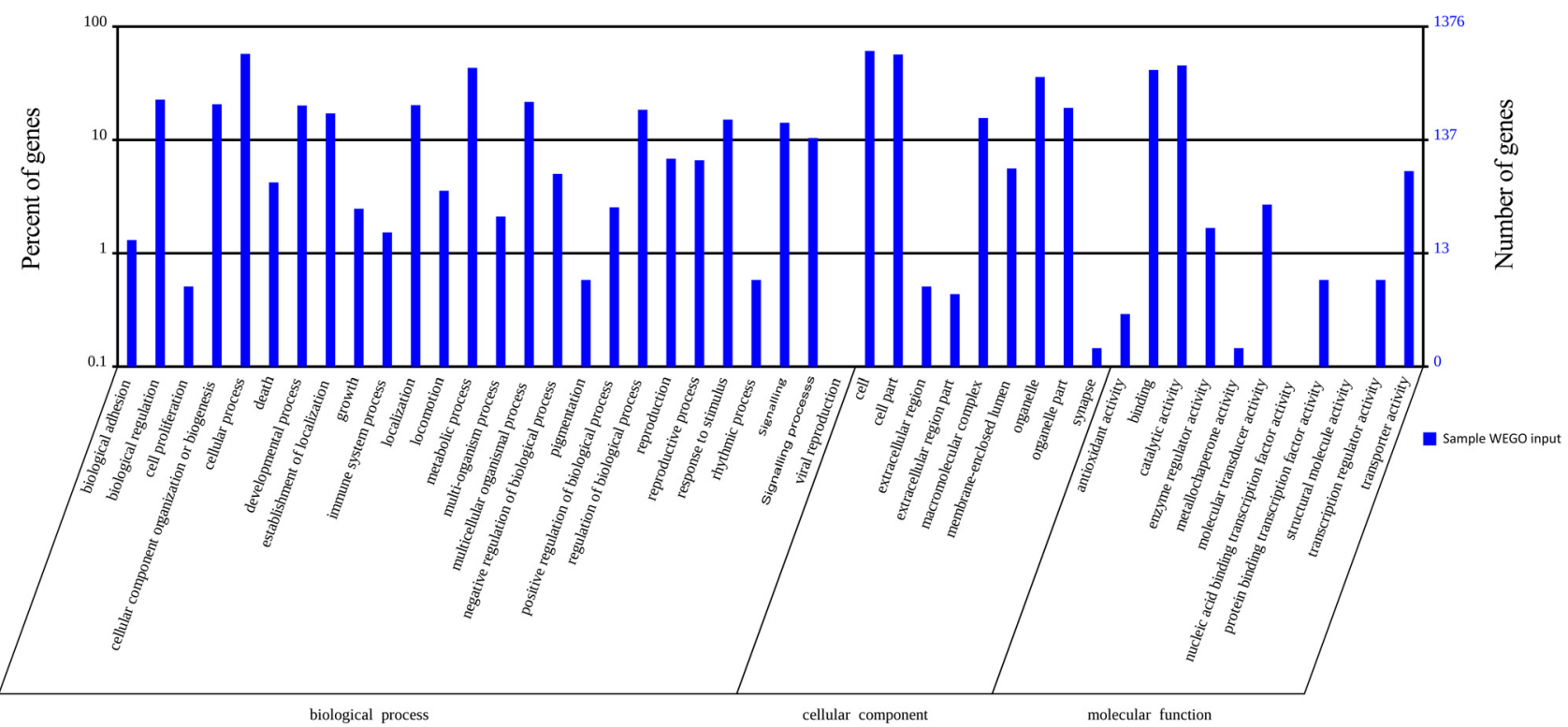

Fig. 3. The gene ontology (GO) distribution of the Macrocentrus cingulum unigenes were annotated by three categories: biological process, cellular component, and molecular function. The left and right $Y$-axis shows the percent and number of genes, and $\mathrm{X}$-axis shows three categories.

After the unigenes were aligned with the COG database we found the following. In total, 1882 unigenes were annotated and classified into 25 clusters of orthologous groups (COG) (Fig. 4). The "General function prediction" cluster group is the largest (277 unigenes, 14.72\%) among the functional classes, followed by "signal transduction mechanism" (232 unigenes, 12.33\%), "post translational modification, protein turnover, chaperones" (210 unigenes, $11.16 \%$ ), "cell motility" (5 unigenes, $0.27 \%$ ) and "nuclear structure" (7 unigenes, 0.37\%).

\section{Annotation of a part of OR and IR genes}

The unigenes with at least one match to ORs and IRs, and with an $E$-value of $1 \mathrm{E}-10^{-5}$ or lower were selectively annotated. After homology search with BLASTX, we obtained 9 OR and 3 IR genes (Table 2). A phylogenetic tree was built with the sequence alignment of the $M$. cingulum 9 OR to 65 OR sequences of others Hymenoptera species (Fig. 5). The ORs of $M$. cingulum are very divergent, and most of them (7 out of 9) have no homologues other hymenopteran species. McinOR2 is clustered with the MmedOR6 and MmedOR8 (Microptilis mediator), and NvitOR87 ( $N$. vitripennis) with $70 \%$ bootstrap cutoff value. McinOR5 also clustered with NvitOR154 and MmedOR5 (Fig. 5) with the same bootstrap cutoff value (70\%).

\section{Expression pattern in different tissues}

We conducted reverse transcription PCR (RT-PCR) analyses in different tissues (antenna, head, leg, thorax and abdomen) of adult males and females to assess the expression of $M$. cingulum OR1 to 9 and IR1 to 3 . All the OR genes were expressed in the female antennae, although OR6 had a low expression. Only OR4 was not expressed in the male antennae, and OR2, OR8, and OR9 were expressed at low levels (Fig. 6). The following had low expressions in other tissues: male head (M.H: OR1, OR5); female head (M.H: OR1, OR4, OR5, OR9); female abdomen (F.Abd: OR1, OR3, OR4, OR8, and OR9); female leg (F.L: OR9). All

Table 2. The part of unigene from Macrocentrus cingulum with similarity to $O R$ and IR genes.

\begin{tabular}{lccclc}
\hline Gene name & Length $(\mathrm{bp})$ & TMHMM $^{*}$ & Accession no. & \multicolumn{1}{c}{ Homology } & $E$-value \\
\hline McinOR1 & 1163 & 3 & KC887063 & Odorant receptor 300 [Nasonia vitripennis] & $2 \mathrm{e}-31$ \\
McinOR2 & 1239 & 2 & KC887064 & Odorant receptor 81 [Nasonia vitripennis] & $6 \mathrm{e}-61$ \\
McinOR3 & 1184 & 5 & KC887065 & Odorant receptor 261 [Nasonia vitripennis] & $8 \mathrm{e}-32$ \\
McinOR4 & 1275 & 3 & KC887066 & Odorant receptor 58 [Apis mellifera] & $1 \mathrm{e}-24$ \\
McinOR5 & 1189 & 2 & KC887067 & Odorant receptor 202 [Nasonia vitripennis] & $5 \mathrm{e}-34$ \\
McinOR6 & 1305 & 1 & KC887068 & Odorant receptor 82 [Nasonia vitripennis] & $1 \mathrm{e}-44$ \\
McinOR7 & 1250 & 4 & KC887069 & Odorant receptor 60 [Nasonia vitripennis] & $1 \mathrm{e}-24$ \\
McinOR8 & 1166 & 4 & KC887070 & Putative odorant receptor 13a [Camponotus floridanus] & $9 \mathrm{e}-37$ \\
McinOR9 & 1140 & 4 & KC887071 & Olfactory receptor 10 [Microplitis mediator] & $9 \mathrm{e}-48$ \\
McinIR1 & 1240 & - & KC887072 & lonotropic receptor 8a [Drosophila melanogaster] & $2 \mathrm{e}-14$ \\
McinIR2 & 1237 & - & KC887073 & Putative ionotropic receptor IR93a, partial [Cydia pomonella] & $3 \mathrm{e}-35$ \\
McinIR3 & 1213 & - & KC887074 & Glutamate receptor, ionotropic kainate 2 [Apis mellifera] & $4 \mathrm{e}-21$ \\
\hline
\end{tabular}

* Transmembrane Helices Hidden Markov Model (protein topology). 

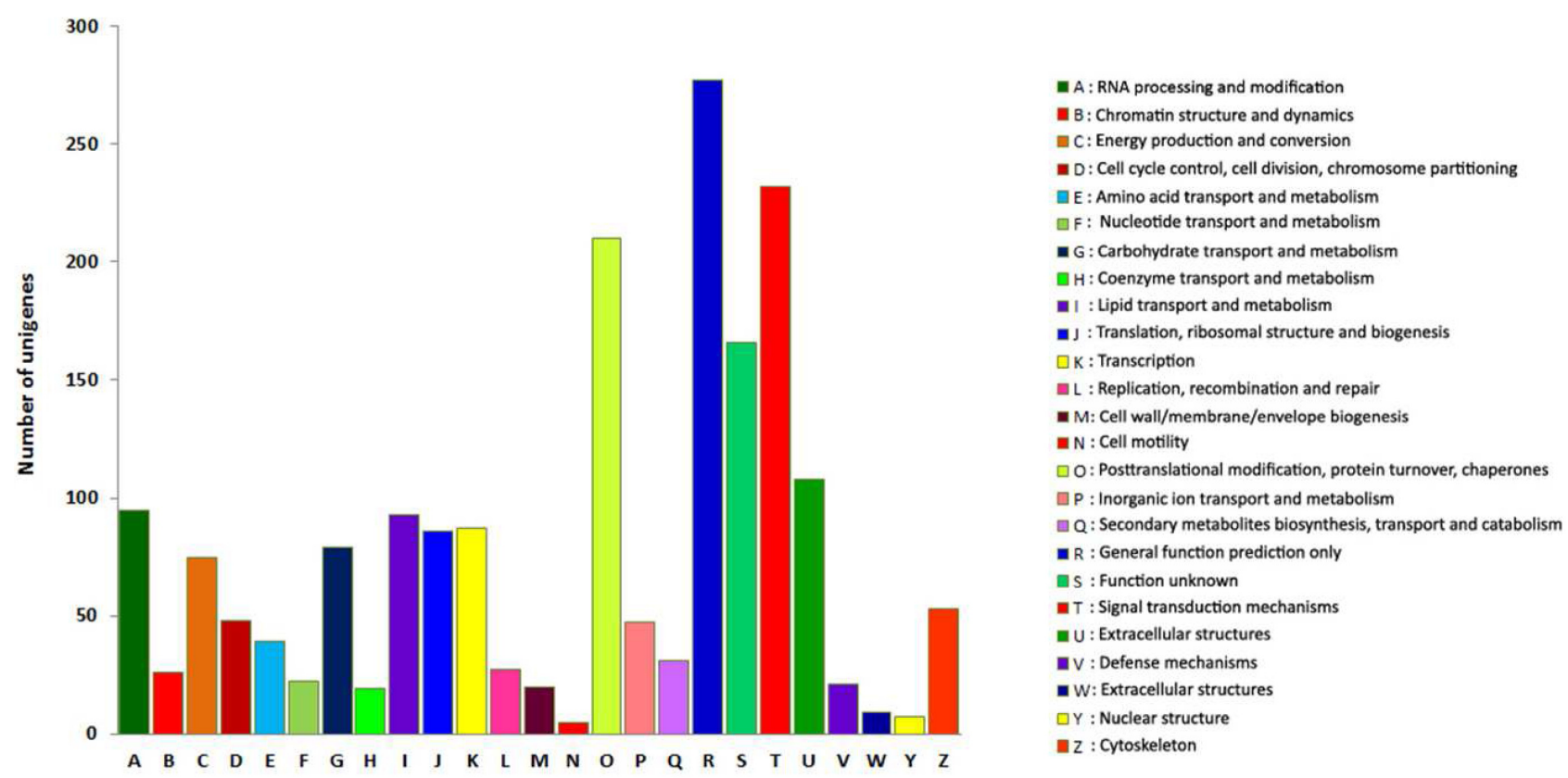

Fig. 4. The clusters of orthologous groups (COG) classification of the unigenes fell into 25 categories.

the IR genes (IR1, IR2, and IR3) expressed in the male antennae and only IR1 expressed (lightly) in the female antennae (Fig. 7). For the other tissues, only IR2 had a high expression, in this case in the male leg (M.L). Low IR gene expressions were found in other tissues: female thorax (F.T: IR2 and IR3); female abdomen (F.Abd: IR2); male thorax (M.T: IR3) (Fig. 7).

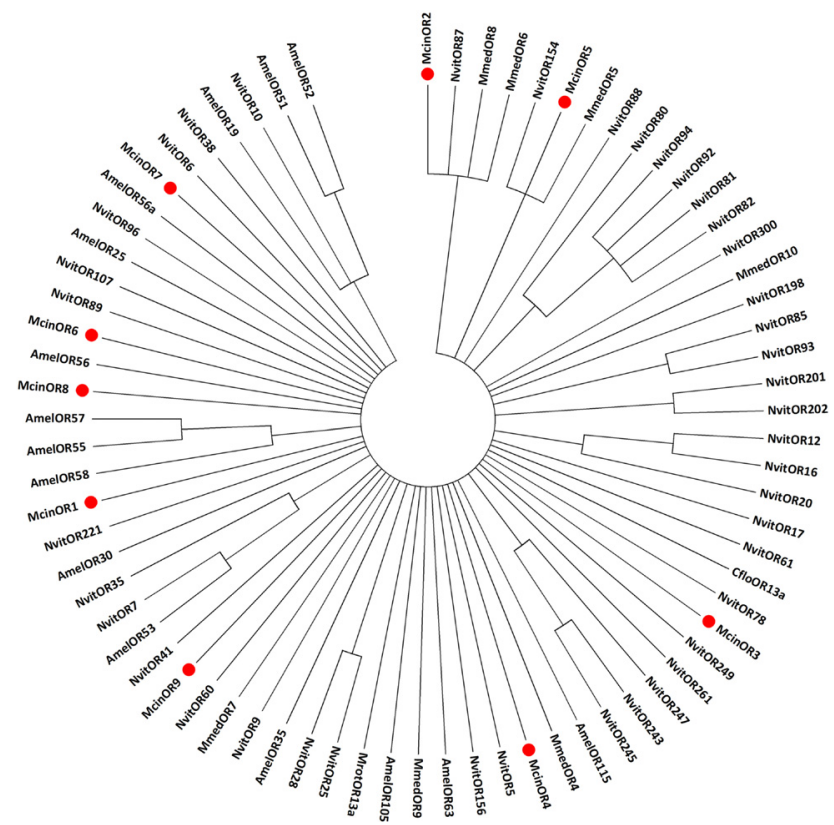

Fig. 5. Phylogenetic tree of annotated amino acids of ORs among Macrocentrus cingulum and other hymenoptera wasp. This tree was constructed from the multiple alignments using MEGA 4.0 software, generated with 1,000 bootstrap replicated samples using the neighbor-joining method, and presented with $70 \%$ cut-off bootstrap value. GenBank accession numbers of the 9 OR are listed in Table 2. Accession numbers of the other insect ORs are listed in Table S1.

\section{DISCUSSION}

We constructed a cDNA library from the antennae of the polyembryonic endoparasitoid, $M$. cingulum and using a bioinformatics approach we categorized the function of many of the genes. The BLASTx annotation of $M$. cingulum cDNA library unigene sequences revealed that 727 of 3160 unigenes $(23.00 \%)$ did not have homologous sequences to other insect or non-insect species, which suggests that these genes may be insect specific. Additionally, we found that $1.42 \%$ of the genes were homologous to non-insect species but had no homology with genome sequences of D. melanogaster, Bombyx mori or Acyrthosiphon pisum (The International Aphid Genomics, 2010). Whether these sequences are $M$. cingulum specific or are non-coding genes needs to be further investigated.

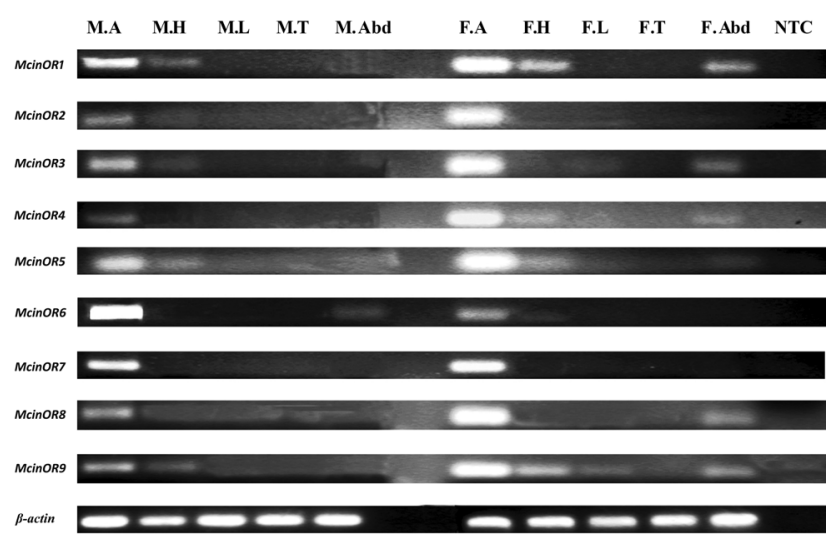

Fig. 6. Tissue and sex specific expression pattern of ORs genes in Macrocentrus cingulum adults. M.A - male antennae, M.H - male head, M.L - male leg, M.T - male thorax, M. Abd - male abdomen, F.A - female antennae, F.H - female head, F.L - female leg, F.T female thorax, F. Abd - female abdomen, NTC - Non tissue control and $\beta$ actin - reference gene of $M$. cingulum. 


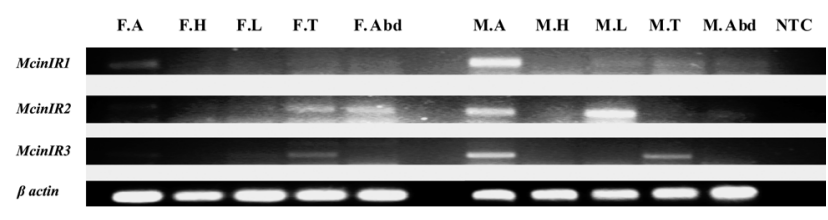

Fig. 7. Tissue and sex specific expression pattern of IRs genes in Macrocentrus cingulum adults. F.A - female antennae, F.H - female head, F.L - female leg, F.T - female thorax, F. Abd - female abdomen, M.A - male antennae, M.H - male head, M.L - male leg, M.T - male thorax, M. Abd - male abdomen, NTC - Non tissue control and $\beta$ actin - reference gene of $M$. cingulum.

Only a few OR genes were identified with the homologue based strategy, with a focus on closely related species (Liu et al., 2012). The most abundant genes in the antennal cDNA libraries were those encoding ORs, suggesting their high expression in $M$. cingulum antennae, which was confirmed by RT-PCR (Fig. 6). The parasitoid wasp $N$. vitripennis, has $301 \mathrm{OR}$ genes, of which 225 are intact genes and 76 are pseudogenes (Robertson et al., 2010). The honey bee $A$. mellifera genome analyses revealed a major expansion of the OR family to 170 genes (Robertson \& Wanner, 2006). The silk moth, B. mori has approximately 60 ORs (Wanner et al., 2007; The International Silkworm Genome, 2008). D. melanogaster and Anopheles gambiae, have 62 and 79 ORs, respectively (Robertson \& Wanner, 2006). Automated genome annotations of $C$. floridanus and $H$. saltator revealed about 100 ORs (Zhou et al., 2012). In this work we identified 9 OR genes in M. cingulum antennae. This is the first study of chemosensory receptors in M. cingulum. Many of the known ORs were not found in our EST data, for which we offer the following explanation: first, we only sequenced the antennal cDNA library of these wasps. Some ORs might be specifically expressed at different developmental stages or in other olfactory tissues. Second, limited ESTs that were sequenced, we reasoned that there are still some ORs not yet to be identified in this wasp. However, the number of identified ORs (9) in M. cingulum is higher than the six found for Cotesia vestalis from the transcriptome pyrosequencing (Nishimura et al., 2012) but less than $M$. mediator, in which 13 ORs were submitted to NCBI. The OR genes might enable the wasp to sense the wide range of pheromones, floral scents, HIPVs and other olfactory cues (Nishimura et al., 2012). Our study is the first to investigate the genetics of chemosensory receptors of M. cingulum, but for more comprehensive analysis, transcriptomic/genomic analyses of this species should be conducted.

All the 9 ORs were specifically expressed in antennae. Experiments were conducted to identify ORs with differential expression patterns between male and female because they might perform specific functions in each gender. The RT-PCR showed that only OR6 was more highly expressed in the male than female antennae but OR7 was expressed in the same level in both the male and female antennae, the other seven were highly expressed in the female than male antennae. The OR1, OR3, OR8, and OR9 were expressed in the female abdomen perhaps due to the presence of some sensilla in the ovipositor sheath. Also, OR1 was expressed at a low level in the male and female head.

IRs as chemosensory receptors have recently been discovered in D. melanogaster (Benton et al., 2009) followed by other species through genome annotation (Croset et al. 2010). In D. melanogaster, 66 IRs were identified (Benton et al., 2009) and 12 were reported in the antennae of $S$. littoralis (Olivier et al., 2011). In the A. mellifera and $N$. vitripennis genomes, 10 IRs genes were found for each (Croset et al., 2010), but the Linepithema humile genome had 32 IR genes (Smith et al., 2011). The two ants, C. floridanus and H. saltator, had 31 and 23 IR genes, respectively (Zhou et al., 2012). In the M. cingulum cDNA library we found only 3 IR genes, including one (IR93a) that appears to be orthologous to conserved IRs in other insect genomes that are expressed in olfactory organs. This is the first report of IRs in M. cingulum. Unlike ORs, the expression of the IRs was higher in the male than female antennae. The antenna specific IR1 was highly expressed in male antenna and IR3 was expressed in the antennae and thorax of both sexes. The IR2 was expressed in different tissues of male and female, but highly expressed in the male legs than antenna.

In summary, we conducted a preliminary study of olfactory gene expression analysis in $M$. cingulum through the construction and annotation of the antennal cDNA library. We identified for the first time 9 OR and 3 IR genes from M. cingulum. Our finding provides a foundational knowledge to understand the chemoreception mechanisms of this wasp. Future studies should characterize the function of these olfactory receptors with corn and $O$. furnacalis volatiles.

ACKNOWLEDGEMENTS. This work was supported by Special Fund for Agro-scientific Research in the Public Interest (201303026) and China Agriculture Research System (CARS02). The authors gratefully acknowledge R.L. Hellmich, USDAARS, Corn Insects and Crop Genetics Research Unit for comments and suggestions on this manuscript.

\section{REFERENCES}

Ahmed T., Zhang T., Wang Z., He K. \& Bai S. 2013: Morphology and ultrastructure of antennal sensilla of Macrocentrus cingulum Brischke (Hymenoptera: Braconidae) and their probable functions. - Micron 50: 35-43.

Altschul S.F., Madden T.L., Schäffer A.A., Zhang J., Zhang Z., Miller W. \& LipMan D.J. 1997: Gapped BLAST and PSIBLAST: a new generation of protein database search programs. — Nucl. Acids Res. 25: 3389-3402.

Andersson M., Grosse-Wilde E., Keeling C., Bengtsson J., Yuen M.M., Li M., Hillbur Y., Bohlmann J., Hansson B. \& SCHLYTER F. 2013: Antennal transcriptome analysis of the chemosensory gene families in the tree killing bark beetles, Ips typographus and Dendroctonus ponderosae (Coleoptera: Curculionidae: Scolytinae). — BMC Genomics 14: 198.

Bengtsson J.M., Trona F., Montagné N., Anfora G., Ignell R., WitzGall P. \& JACQUin-Joly E. 2012: Putative chemosensory receptors of the codling moth, Cydia pomonella, identified by antennal transcriptome analysis. - PLOS ONE 7: e31620.

Benton R., Sachse S., Michnick S.W. \& Vosshall L.B. 2006: Atypical membrane topology and heteromeric function of Drosophila odorant receptors in vivo. - PLoS Biol. 4: e20. 
Benton R., Vannice K.S., Gomez-Diaz C. \& Vosshall L.B. 2009: Variant ionotropic glutamate receptors as chemosensory receptors in Drosophila. - Cell 136: 149-162.

Clyne P.J., Warr C.G., Freeman M.R., Lessing D., Kim J. \& CARLSON J.R. 1999: A novel family of divergent seven-transmembrane proteins: candidate odorant receptors in Drosophila. - Neuron 22: 327-338

Conesa A., Götz S., García-Gómez J.M., Terol J., Talón M. \& RoBLEs M. 2005: Blast2GO: a universal tool for annotation, visualization and analysis in functional genomics research. Bioinformatics 21: 3674-3676.

Couto A., Alenius M. \& Dickson B.J. 2005: Molecular, anatomical, and functional organization of the Drosophila olfactory system. - Curr. Biol. 15: 1535-1547.

Croset V., Rytz R., Cummins S.F., Budd A., Brawand D., Kaessmann H., Gibson T.J. \& Benton R. 2010: Ancient protostome origin of chemosensory ionotropic glutamate receptors and the evolution of insect taste and olfaction. - PLoS Genet. 6 : e1001064.

EDWARDS O.R. \& HopPER K.R. 1999: Using superparasitism by a stem borer parasitoid to infer a host refuge. - J. Ecol. Entomol. 24: 7-12.

GAO Q. \& CHESS A. 1999: Identification of candidate Drosophila olfactory receptors from genomic DNA sequence. - Genomics 60: 31-39.

Gong D.P., Zhang H.J., Zhao P., Lin Y., Xia Q.Y. \& Xiang Z.H. 2007: Identification and expression pattern of the chemosensory protein gene family in the silkworm, Bombyx mori. $-J$. Insect Biochem. Mol. Biol. 37: 266-277.

Gong D.P., Zhang H.J., Zhao P., XIA Q.Y. \& Xiang Z.H. 2009: The odorant binding protein gene family from the genome of silkworm, Bombyx mori. - BMC Genomics 10: 332.

Götz S., Garcia-Gómez J.M., Terol J., Williams T.D., Nagaraj S.H., Nueda M.J., Robles M., Talón M., Dopazo J.N. \& ConeSA A. 2008: High-throughput functional annotation and data mining with the Blast2GO. - Nucl. Acids Res. 36: 3420-3435.

Grosse-Wilde E., Kuebler L.S., Bucks S., Vogel H., Wicher D. \& Hansson B.S. 2011: Antennal transcriptome of Manduca sexta. - Proc. Natl. Acad. Sci. USA 108: 7449-7454.

Howlett N., Dauber K., Shukla A., Morton B., Glendinning J., Brent E., Gleason C., Islam F., Izquierdo D., Sanghavi S., Afroz A., Aslam A., Barbaro M., Blutstein R., Borovka M. Desire B., Elikhis A., Fan Q., Hoffman K., Huang A., Keefe D., Lopatin S., Miller S., Patel P., Rizzini D., Robinson A., Rokins K., Turlik A. \& Mansfield J. 2012: Identification of chemosensory receptor genes in Manduca sexta and knockdown by RNA interference. - BMC Genomics 13: 211.

Hu J., ZHU X. \& Fu W. 2003: Passive evasion of encapsulation in Macrocentrus cingulum Brischke (Hymenoptera: Braconidae), a polyembryonic parasitoid of Ostrinia furnacalis Guenée (Lepidoptera: Pyralidae). — J. Insect Physiol. 49: 367-375.

Larsson M.C., Domingos A.I., Jones W.D., Chiappe M.E., Amrein H. \& Vosshall L.B. 2004: Or83b encodes a broadly expressed odorant receptor essential for Drosophila olfaction. - Neuron 43: 703-714.

Liu Y., Gu S., Zhang Y., Guo Y. \& Wang G. 2012: Candidate olfaction genes identified within the Helicoverpa armigera antennal transcriptome. - PLOS ONE 7: e48260.

Nishimura O., Brillada C., Yazawa S., Maffei M.E. \& Arimura G. 2012: Transcriptome pyrosequencing of the parasitoid wasp Cotesia vestalis: genes involved in the antennal odorant-sensory system. - PLOS ONE 7: e50664.

NordLund D.A., Lewis W.J. \& Altieri M.A. 1988: Influence of plant produced allelochemicals on host-prey selection behavior of entomophagous insects. In Barbosa P. \& Letourneau D.K. (eds): Novel Aspects of Insect-Plant Interaction. Wiley, New York, pp. 65-90.

Ochieng S.A., Park K.C., Zhu J.W. \& BaKer T.C. 2000: Functional morphology of antennal chemoreceptors of the parasitoid Microplitis croceipes (Hymenoptera: Braconidae). Arthr. Star. Dev. 29: 231-240.

Olivier V., Monsempes C., François M.C., Poivet E. \& JacquinJOLY E. 2011: Candidate chemosensory ionotropic receptors in a Lepidoptera. - Insect Mol. Biol. 20: 189-199.

PARKER H.L. 1931: Macrocentrus gifuensis Ashmead, a polyembrinic braconid parasite in the European corn borer. - Tech. Bull. 230: 1-61.

RoBERTSON H.M. \& WANNER K.W. 2006: The chemoreceptor superfamily in the honey bee, Apis mellifera: expansion of the odorant, but not gustatory, receptor family. — Genome Res. 16: $1395-1403$.

Robertson H.M., Gadau J. \& Wanner K.W. 2010: The insect chemoreceptor superfamily of the parasitoid jewel wasp Nasonia vitripennis. - Insect Mol. Biol. 19: 121-136.

Shiojiri K., Takabayashi J., Yano S. \& Takafuji A. 2000: Flight response of parasitoids toward plant-herbivore complexes: A comparative study of two parasitoid-herbivore systems on cabbage plants. - Appl. Entomol. Zool. 35: 87-92.

Smith C.D., Zimin A., Holt C., Abouheif E., Benton R., Cash E., Croset V., Currie C.R., Elhaik E., Elsik C.G., Fave M.J., Fernandes V., Gadau J., Gibson J.D., Graur D., Grubbs K.J., Hagen D.E., HelmKampf M., Holley J.A., Hu H., Viniegra A.S.I., Johnson B.R., Johnson R.M., KhILA A., Kim J.W., LAIRD J., Mathis K.A., Moeller J.A., MuÑoz-Torres M.C., MurPhy M.C., Nakamura R., Nigam S., Overson R.P., Placek J.E., Rajakumar R., Reese J.T., Robertson H.M., Smith C.R., Suarez A.V., Suen G., Suhr E.L., Tao S., Torres C.W., van Wilgenburg E., Viljakainen L., Walden K.K.O., Wild A.L., Yandell M., Yorke J.A. \& Tsutsui N.D. 2011: Draft genome of the globally widespread and invasive Argentine ant (Linepithema humile). - Proc. Natl. Acad. Sci. USA 108: 5673-5678.

Su C.Y., Menuz K. \& CARLSON J.R. 2009: Olfactory perception: receptors, cells, and circuits. - Cell 139: 45-59.

Tamura K., Dudley J., Nei M. \& Kumar S. 2007: MEGA4: Molecular Evolutionary Genetics Analysis (MEGA) software version 4.0. - Mol. Biol. Evol. 24: 1596-1599.

Tatusov R.L., Galperin M.Y., Natale D.A. \& Koonina E.V. 2000: The COG database: a tool for genome-scale analysis of protein functions and evolution. - Nucl. Acids Res. 28: 33-36.

The International Aphid Genomics C. 2010: Genome sequence of the pea aphid Acyrthosiphon pisum. - PLoS Biol. 8: e1000313.

The International Silkworm Genome C. 2008: The genome of a lepidopteran model insect, the silkworm Bombyx mori. - Insect Biochem. Mol. Biol. 38: 1036-1045.

Vosshall L.B., Amrein H., Morozov P.S., Rzhetsky A. \& Axel R. 1999: A spatial map of olfactory receptor expression in the Drosophila antenna. - Cell 96: 725-736.

Wanner K., Anderson A., Trowell S., Theilmann D., Robertson H. \& Newcomb R. 2007: Female-biased expression of odourant receptor genes in the adult antennae of the silkworm, Bombyx mori. - Insect Mol. Biol. 16: 107-119.

Watanabe C. 1967: Further revision of the genus Macrocentrus Curtis in Japan, with descriptions of two new species (Hymenoptera, Braconidae). - Insecta Matsum. 30: 1-16.

Ye J., Fang L., Zheng H., Zhang Y., Chen J., Zhang Z., Wang J., LI S., Li R., Bolund L. \& WANG J. 2006: WEGO: a web tool for plotting GO annotations. - Nucl. Acids Res. 34: W293-W297. 
Zhou D.R., WANG Y.Y., Liu B.L. \& Ju Z.L. 1980: Research on the reproduction of $O$. furnacalis in a large quantity: an artificial diet and its improvement. - Acta Phytophyl. Sin. 7: 113-122.

Zhou X., Slone J.D., Rokas A., Berger S.L., Liebig J., Ray A., Reinberg D. \& Zwiebel L.J. 2012: Phylogenetic and transcriptomic analysis of chemosensory receptors in a pair of divergent ant species reveals sex-specific signatures of odor coding. PLoS Genet. 8: e1002930.

Received July 10, 2015; revised and accepted September 21, 2015 Published online January 11, 2016
Table S1. GenBank accession numbers of OR of different hymenopteran insects.

\begin{tabular}{|c|c|c|}
\hline Species name & OR gene no. & GB accession no. \\
\hline Apis melifera & $\begin{array}{l}\text { AmelOR6 } \\
\text { AmelOR19 } \\
\text { AmelOR25 } \\
\text { AmelOR30 } \\
\text { AmelOR35 } \\
\text { AmelOR51 } \\
\text { AmelOR52 } \\
\text { AmelOR53 } \\
\text { AmelOR55 } \\
\text { AmelOR56 } \\
\text { AmelOR56a } \\
\text { AmelOR57 } \\
\text { AmelOR58 } \\
\text { AmelOR63 } \\
\text { AmelOR105 } \\
\text { AmelOR115 }\end{array}$ & $\begin{array}{l}\text { NP_001177432.1 } \\
\text { NP_001229895.1 } \\
\text { NP_001229896.1 } \\
\text { NP_001229899.1 } \\
\text { NP_001229900.1 } \\
\text { NP_001229905.1 } \\
\text { NP_001229906.1 } \\
\text { NP_001229907.1 } \\
\text { NP_001229908.1 } \\
\text { NP_001229909.1 } \\
\text { XP_001122191.2 } \\
\text { NP_001229910.1 } \\
\text { NP_001229911.1 } \\
\text { NP_001229913.1 } \\
\text { NP_001229915.1 } \\
\text { NP_001229918.1 }\end{array}$ \\
\hline Camponotus floridanus & CfloOR13a & XM_011265538.1 \\
\hline Microptilis mediator & $\begin{array}{l}\text { MmedOR4 } \\
\text { MmedOR5 } \\
\text { MmedOR6 } \\
\text { MmedOR7 } \\
\text { MmedOR8 } \\
\text { MmedOR9 } \\
\text { MmedOR10 } \\
\end{array}$ & $\begin{array}{l}\text { AGG17937.1 } \\
\text { AGG17938.1 } \\
\text { AGG17939.1 } \\
\text { AGG17940.1 } \\
\text { AGG17941.1 } \\
\text { AGG17943.1 } \\
\text { AGG17942.1 }\end{array}$ \\
\hline Megachile rotundata & MrotOR13a & XM_003700884.1 \\
\hline Nasonia vitripennis & $\begin{array}{l}\text { NvitOR5 } \\
\text { NvitOR7 } \\
\text { NvitOR9 } \\
\text { NvitOR10 } \\
\text { NvitOR12 } \\
\text { NvitOR16 } \\
\text { NvitOR17 } \\
\text { NvitOR20 } \\
\text { NvitOR25 } \\
\text { NvitOR28 } \\
\text { NvitOR35 } \\
\text { NvitOR38 } \\
\text { NvitOR41 } \\
\text { NvitOR60 } \\
\text { NvitOR61 } \\
\text { NvitOR78 } \\
\text { NvitOR80 } \\
\text { NvitOR81 } \\
\text { NvitOR82 } \\
\text { NvitOR85 } \\
\text { NvitOR87 } \\
\text { NvitOR93 }\end{array}$ & $\begin{array}{l}\text { NP_001177431.1 } \\
\text { NP_001177433.1 } \\
\text { NP_001177435.1 } \\
\text { NP_001177472.1 } \\
\text { NP_001177468.1 } \\
\text { NP_001177472.1 } \\
\text { NP_001177473.1 } \\
\text { NP_001177475.1 } \\
\text { NP_001177480.1 } \\
\text { NP_001177483.1 } \\
\text { NP_001177486.1 } \\
\text { NP_001177489.1 } \\
\text { NP_001164391.1 } \\
\text { NP_001177501.1 } \\
\text { NP_001177502.1 } \\
\text { NP_001177510.1 } \\
\text { NP_001164396.1 } \\
\text { NP_001164394.1 } \\
\text { NP_001164395.1 } \\
\text { NP_001164398.1 } \\
\text { NP_001177513.1 } \\
\text { NP_001177516.1 }\end{array}$ \\
\hline
\end{tabular}

